\title{
MORREY SPACES ARE EMBEDDED BETWEEN WEAK MORREY SPACES AND STUMMEL CLASSES
}

\author{
NICKY K. TUMALUN ${ }^{1}$ AND HENDRA GUNAWAN ${ }^{2}$ \\ Faculty of Mathematics and Natural Sciences, \\ Institut Teknologi Bandung, Bandung 40132, Indonesia \\ ${ }^{1}$ nickytumalun@yahoo.co.id, ${ }^{2}$ hgunawan@math.itb.ac.id
}

\begin{abstract}
In this paper, we show that the Morrey spaces $L^{1,\left(\frac{\lambda}{p}-\frac{n}{p}+n\right)}\left(\mathbb{R}^{n}\right)$ are embedded between weak Morrey spaces $w L^{p, \lambda}\left(\mathbb{R}^{n}\right)$ and Stummel classes $S_{\alpha}\left(\mathbb{R}^{n}\right)$ under some conditions on $p, \lambda$ and $\alpha$. More precisely, we prove that $w L^{p, \lambda}\left(\mathbb{R}^{n}\right) \subseteq$ $L^{1,\left(\frac{\lambda}{p}-\frac{n}{p}+n\right)}\left(\mathbb{R}^{n}\right) \subseteq S_{\alpha}\left(\mathbb{R}^{n}\right)$ where $1<p<\infty, 0<\lambda<n$ and $\frac{n-\lambda}{p}<\alpha<n$. We also show that these inclusion relations under the above conditions are proper. Lastly, we present an inequality of Adams' type [1].

Key words and phrases: Morrey spaces, Stummel classes, Adams' type inequality.
\end{abstract}

Abstrak. Pada makalah ini, dibuktikan bahwa ruang Morrey $L^{1,\left(\frac{\lambda}{p}-\frac{n}{p}+n\right)}\left(\mathbb{R}^{n}\right)$ tersisipkan di antara ruang Morrey lemah $w L^{p, \lambda}\left(\mathbb{R}^{n}\right)$ dan kelas Stummel $S_{\alpha}\left(\mathbb{R}^{n}\right)$ untuk $p, \lambda$ dan $\alpha$ tertentu. Persisnya, dibuktikan bahwa $w L^{p, \lambda}\left(\mathbb{R}^{n}\right) \subseteq$ $L^{1,\left(\frac{\lambda}{p}-\frac{n}{p}+n\right)}\left(\mathbb{R}^{n}\right) \subseteq S_{\alpha}\left(\mathbb{R}^{n}\right)$ dengan $1<p<\infty, 0<\lambda<n$ dan $\frac{n-\lambda}{p}<\alpha<n$. Akan dibuktikan pula bahwa hubungan inklusi ini merupakan inklusi sejati. Di akhir makalah, disajikan suatu ketaksamaan tipe Adams [1].

Kata kunci: Ruang Morrey, kelas Stummel, ketaksamaan tipe Adams.

\section{INTRODUCTION}

The notion of Stummel classes was defined in [5, 11]. For $0<\alpha<n$, the Stummel class $S_{\alpha}\left(\mathbb{R}^{n}\right)$ is defined to be the set

$$
S_{\alpha}\left(\mathbb{R}^{n}\right):=\left\{f \in L_{\mathrm{loc}}^{1}\left(\mathbb{R}^{n}\right): \eta_{\alpha} f(r) \searrow 0 \text { for } \quad r \searrow 0\right\},
$$


where

$$
\eta_{\alpha} f(r):=\sup _{x \in \mathbb{R}^{n}} \int_{|x-y|<r} \frac{|f(y)|}{|x-y|^{n-\alpha}} d y .
$$

The $\eta_{\alpha}$ is called the Stummel modulus of $f$. For $\alpha:=2$, the class $S_{2}$ is known as the Kato class or the Stummel-Kato class. The Stummel classes $S_{\alpha}\left(\mathbb{R}^{n}\right)$ have an application in studying the regularity theory of partial differential equation. Such an application can be found in $[2,3,4,6,9]$.

In [11], Ragusa and Zamboni studied the inclusion relation between Morrey spaces and Stummel classes. They proved the following result:

Lemma 1.1. [11] Let $0<\alpha<n$ and $n-\alpha<\gamma<n$. If $f \in L^{1, \gamma}\left(\mathbb{R}^{n}\right)$, then

$$
\eta_{\alpha} f(r) \leq C(n, \alpha, \gamma) r^{\gamma-n+\alpha}\|f\|_{L^{1, \gamma}\left(\mathbb{R}^{n}\right)} .
$$

Therefore, $L^{1, \gamma}\left(\mathbb{R}^{n}\right) \subseteq S_{\alpha}\left(\mathbb{R}^{n}\right)$.

Note here that, the Morrey space $L^{1, \gamma}\left(\mathbb{R}^{n}\right)$ is the collection of all functions $f \in L_{\text {loc }}^{1}\left(\mathbb{R}^{n}\right)$ for which

$$
\|f\|_{L^{1, \gamma}\left(\mathbb{R}^{n}\right)}:=\sup _{x \in \mathbb{R}^{n}, r>0} \frac{1}{r^{\gamma}} \int_{|x-y|<r}|f(y)| d y<\infty, \quad 0<\gamma<n .
$$

For the case $\alpha:=2$, the Lemma 1.1 was proved by Di Fazio in [6]. There are also some studies between generalized Stummel classes and generalized Morrey spaces that can be found in $[7,8,12]$.

We need to write down the definition of weak Morrey spaces since this article deals with them. For $1 \leq p<\infty$ and $0 \leq \lambda \leq n$, we define $w L^{p, \lambda}\left(\mathbb{R}^{n}\right)$ the weak Morrey space to be the set of all functions $f \in w L_{\mathrm{loc}}^{p}\left(\mathbb{R}^{n}\right)$ for which

$$
\|f\|_{w L^{p, \lambda}\left(\mathbb{R}^{n}\right)}:=\sup _{x \in \mathbb{R}^{n}, r>0} r^{-\lambda / p}\|f\|_{w L^{p}(B(x, r))}<\infty,
$$

where

$$
\|f\|_{w L^{p}(B(x, r))}:=\sup _{t>0} t(\mu(\{y \in B(x, r):|f(y)|>t\}))^{\frac{1}{p}},
$$

with $\mu$ being the Lebesgue measure on $\mathbb{R}^{n}$ and $B(x, r):=\left\{y \in \mathbb{R}^{n}:|x-y|<r\right\}$.

In this paper, we show that there are Morrey spaces 'between' some weak Morrey spaces and Stummel classes, in the sense of proper inclusions. Since the Morrey spaces and Stummel classes are applied in studying regularity properties of some partial differential equation (see $[1,3,10]$ ), the inclusion properties of these spaces (classes) are useful to study. We also present an inequality of Adams' type [1] as our last result.

\section{THE INCLUSION OF WEAK MORREY SPACES, MORREY SPACCES, AND STUMMEL CLASSES}

The first proposition is about the inclusion relation between weak Morrey spaces and Morrey spaces. 
Proposition 2.1. Let $1<p<\infty$ and $0<\lambda<n$. If $f \in w L^{p, \lambda}\left(\mathbb{R}^{n}\right)$, then

$$
\|f\|_{\left.L^{1,\left(\frac{\lambda}{p}-\frac{n}{p}+n\right.}\right)_{\left(\mathbb{R}^{n}\right)}} \leq C(n, \lambda, p)\|f\|_{w L^{p, \lambda}\left(\mathbb{R}^{n}\right)}^{p} .
$$

Therefore, $w L^{p, \lambda}\left(\mathbb{R}^{n}\right) \subseteq L^{1,\left(\frac{\lambda}{p}-\frac{n}{p}+n\right)}\left(\mathbb{R}^{n}\right)$.

Proof. Take any $f \in w L^{p, \lambda}\left(\mathbb{R}^{n}\right), x \in \mathbb{R}^{n}$, and $r>0$. For every $\sigma \in \mathbb{R}$, we have

$$
\begin{aligned}
\int_{0}^{\infty} \mu(\{y \in B(x, r):|f(y)|>t\}) d t \leq & \int_{0}^{(\mu(B(x, r)))^{\sigma}} \mu(B(x, r)) d t \\
& +\|f\|_{w L^{p, \lambda}\left(\mathbb{R}^{n}\right)}^{p} \int_{(\mu(B(x, r)))^{\sigma}}^{\infty} \frac{r^{\lambda}}{t^{p}} d t \\
= & (\mu(B(x, r)))^{\sigma+1} \\
& +C(p)\|f\|_{w L^{p, \lambda\left(\mathbb{R}^{n}\right)}}^{p}(\mu(B(x, r)))^{\sigma(1-p)} r^{\lambda} .
\end{aligned}
$$

Let $\sigma:=\frac{\lambda}{n p}-\frac{1}{p}$ and $\beta:=\frac{1}{p}(\lambda-n)+n$. Then $n(\sigma+1)=n \sigma(1-p)+\lambda=\beta$. By (1), we obtain

$$
\begin{aligned}
\frac{1}{r^{\left(\frac{\lambda}{p}-\frac{n}{p}+n\right)}} \int_{|x-y|<r}|f(y)| d y & =\frac{1}{r^{\beta}} \int_{|x-y|<r}|f(y)| d y \\
& =\frac{1}{r^{\beta}} \int_{0}^{\infty} \mu(\{y \in B(x, r):|f(y)|>t\}) d t \\
& \leq C(n, \lambda, p)\|f\|_{w L^{p, \lambda}\left(\mathbb{R}^{n}\right)}^{p}
\end{aligned}
$$

for every $x \in \mathbb{R}^{n}$ and $r>0$. From (2), the conclusion follows.

From the Proposition 2.1 and Lemma 1.1, we have the following corollary.

Corollary 2.2. Let $1<p<\infty, 0<\lambda<n$, and $\frac{n-\lambda}{p}<\alpha<n$. If $f \in w L^{p, \lambda}\left(\mathbb{R}^{n}\right)$, then

$$
\|f\|_{\left.L^{1,\left(\frac{\lambda}{p}-\frac{n}{p}+n\right.}\right)_{\left(\mathbb{R}^{n}\right)}} \leq C(n, \lambda, p)\|f\|_{w L^{p, \lambda}\left(\mathbb{R}^{n}\right)}^{p} .
$$

If $f \in L^{1,\left(\frac{\lambda}{p}-\frac{n}{p}+n\right)}\left(\mathbb{R}^{n}\right)$, then

$$
\eta_{\alpha} f(r) \leq C(n, \alpha, \lambda, p) r^{\frac{1}{p}(\lambda-n)+\alpha}\|f\|_{L^{1,\left(\frac{\lambda}{p}-\frac{n}{p}+n\right)}\left(\mathbb{R}^{n}\right)} .
$$

Therefore, $w L^{p, \lambda}\left(\mathbb{R}^{n}\right) \subseteq L^{1,\left(\frac{\lambda}{p}-\frac{n}{p}+n\right)}\left(\mathbb{R}^{n}\right) \subseteq S_{\alpha}\left(\mathbb{R}^{n}\right)$.

In the next example, we show that the inclusion relation which was shown in Lemma 1.1 is proper. Thus, the later inclusion in Corollary 2.2 above is also proper.

Example 2.3. Let $0<\alpha<n$ and $n-\alpha<\gamma<n$. Define $f: \mathbb{R}^{n} \rightarrow \mathbb{R}$ by the formula

$$
f(y):=\frac{\chi_{B}(y)}{|y|^{\alpha}|\ln (|y|)|^{2}}, \quad B:=B(0, \delta), \quad y \in \mathbb{R}^{n},
$$

where $\delta:=e^{\frac{-2}{\alpha}}$. Then $f \in S_{\alpha}\left(\mathbb{R}^{n}\right) \backslash L^{1, \gamma}\left(\mathbb{R}^{n}\right)$. 
To verify the example, we first show that $f \in S_{\alpha}\left(\mathbb{R}^{n}\right)$. Since the function $f$ is radial and non-increasing, the Stummel modulus of $f$ is attained at the origin. Given $r>0$, let $\epsilon:=\min \{r, \delta\}$. We have,

$$
\eta_{\alpha} f(r)=\int_{|y|<r} \frac{\chi_{B}(y)}{|y|^{n}|\ln (|y|)|^{2}} d y=\int_{|y|<\epsilon} \frac{1}{|y|^{n}|\ln (|y|)|^{2}} d y .
$$

By switching to polar coordinate, we obtain

$$
\int_{|y|<\epsilon} \frac{1}{|y|^{n}|\ln (|y|)|^{2}} d y=\frac{C(n)}{-\ln (\epsilon)} .
$$

Therefore,

$$
\eta_{\alpha} f(r)=\frac{C(n)}{-\ln (\epsilon)} .
$$

By the definition of $\epsilon$, for every $0<r<\delta$, we have

$$
\eta_{\alpha} f(r)=C(n)\left(\frac{1}{-\ln (r)}\right) \searrow 0 \text { for } r \searrow 0 .
$$

Thus, $f \in S_{\alpha}\left(\mathbb{R}^{n}\right)$.

Next, using the fact that $1 / t^{\alpha}|\ln (t)|^{2}$ is decreasing on $(0, \delta)$ and the condition $n-\alpha<\gamma$, one may observe that $f \notin L^{1, \gamma}\left(\mathbb{R}^{n}\right)$.

\section{AN ADAMS' TYPE INEQUALITY}

In his paper [1], D. Adams proved the following inequality:

$$
\begin{gathered}
\left(\int_{\mathbb{R}^{n}}|u(x)|^{q} V(x) d x\right)^{\frac{1}{q}} \leq C(p, \gamma, n)\|V\|_{L^{1, \gamma}\left(\mathbb{R}^{n}\right)}\|\nabla u\|_{L^{p}\left(\mathbb{R}^{n}\right)} \\
\forall u \in C_{0}^{\infty}\left(\mathbb{R}^{n}\right), \quad q:=\frac{p \gamma}{n-p}, \quad 1<p<n,
\end{gathered}
$$

where $V$ is a non-negative function in the Morrey space $L^{1, \gamma}\left(\mathbb{R}^{n}\right)$ and $\gamma>n-p$.

Our purpose in this section is to establish an inequality similar to (3). To do so, we need to recall the $S_{\alpha, \varphi}$ class that was introduced in [11].

Let $1<\alpha<n$ and $\varphi:(0, \infty) \rightarrow(0, \infty)$ be a nondecreasing continuous function such that $\lim _{t \rightarrow 0} \varphi(t)=0$. We say that $V: \mathbb{R}^{n} \rightarrow \mathbb{R}$ belongs to the class $S_{\alpha, \varphi}$ if there exists a non decreasing function $\xi_{V}:(0, \infty) \rightarrow(0, \infty)$ with $\lim _{r \rightarrow 0} \xi_{V}(r)=0$ such that

$$
\sup _{x \in \mathbb{R}^{n}} \int_{|x-y|<r} \frac{|V(y)|}{|x-y|^{n-\alpha} \varphi(|x-y|)} d y \leq \xi_{V}(r) .
$$

The following lemma was proved in [11]. It gives a sufficient condition for a function in $S_{\alpha}\left(\mathbb{R}^{n}\right)$ to belong to an appropriate $S_{\alpha, \varphi}$ class.

Lemma $3.1([11])$. Let $V \in S_{\alpha}$ and suppose that there exists $\vartheta \in(0,1)$ such that

$$
\int_{0}^{1} \frac{\left[\eta_{\alpha} V(t)\right]^{1-\vartheta}}{t} d t<\infty .
$$

Then $V \in S_{\alpha,\left[\eta_{\alpha} V\right]^{\vartheta}}$. 
The next theorem is an Adams' type inequality that we obtain.

Theorem 3.2. Let $1<\alpha<n$ and suppose that

$$
\int_{0}^{1} \frac{[\varphi(t)]^{\frac{\alpha^{\prime}}{\alpha}}}{t} d t<\infty
$$

where $\frac{1}{\alpha}+\frac{1}{\alpha^{\prime}}=1$. If $V \in S_{\alpha, \varphi}$, then

$$
\left\{\int_{\mathbb{R}^{n}}|u(x)|^{\alpha}|V(x)| d x\right\}^{\frac{1}{\alpha}} \leq C\left(n, \alpha, \varphi, r_{0}\right)\left[\xi_{V}\left(2 r_{0}\right)\right]^{\frac{1}{\alpha}}\|\nabla u\|_{L^{\alpha}}
$$

for every $u \in C_{0}^{\infty}\left(\mathbb{R}^{n}\right)$ with $\operatorname{supp}(u) \subseteq B\left(x_{0}, r_{0}\right)$.

Proof. Let $x \in B\left(x_{0}, r_{0}\right)$. Since $\int_{0}^{1}[\varphi(t)]^{\frac{\alpha^{\prime}}{\alpha}} t^{-1} d t<\infty$, we have

$$
\int_{B\left(x_{0}, r_{0}\right)} \frac{[\varphi(|x-y|)]^{\frac{\alpha^{\prime}}{\alpha}}}{|x-y|^{n}} d y<\infty
$$

and the value is independent of $x$. By Hölder's inequality, we obtain

$$
\begin{aligned}
\int_{B\left(x_{0}, r_{0}\right)} \frac{|\nabla u(y)|}{|x-y|^{n-1}} d y & \leq\left\{\int_{B\left(x_{0}, r_{0}\right)} \frac{|\nabla u(y)|^{\alpha}}{|x-y|^{n-\alpha} \varphi(|x-y|)} d y\right\}\left\{\int_{B\left(x_{0}, r_{0}\right)} \frac{[\varphi(|x-y|)]^{\frac{\alpha^{\prime}}{\alpha}}}{|x-y|^{n}} d y\right\}^{\frac{1}{\alpha^{\prime}}} \\
& =C\left\{\int_{B\left(x_{0}, r_{0}\right)} \frac{|\nabla u(y)|^{\alpha}}{|x-y|^{n-\alpha} \varphi(|x-y|)} d y\right\}^{\frac{1}{\alpha}},
\end{aligned}
$$

where $C:=C\left(n, \alpha, \varphi, r_{0}\right)$. We now employ the following inequality

$$
|u(x)| \leq C(n) \int_{B\left(x_{0}, r_{0}\right)} \frac{|\nabla u(y)|}{|x-y|^{n-1}} d y
$$

to get

$$
\begin{aligned}
|u(x)|^{\alpha} & \leq C(n, \alpha)\left\{\int_{B\left(x_{0}, r_{0}\right)} \frac{|\nabla u(y)|}{|x-y|^{n-1}} d y\right\}^{\alpha} \\
& \leq C\left(n, \alpha, \varphi, r_{0}\right) \int_{B\left(x_{0}, r_{0}\right)} \frac{|\nabla u(y)|^{\alpha}}{|x-y|^{n-\alpha} \varphi(|x-y|)} d y .
\end{aligned}
$$


Since $B\left(x_{0}, r_{0}\right) \supseteq \operatorname{supp}(u)$, by Tonneli's theorem we obtain

$$
\begin{aligned}
\int_{\mathbb{R}^{n}}|u(x)|^{\alpha}|V(x)| d x & =\int_{B\left(x_{0}, r_{0}\right)}|u(x)|^{\alpha}|V(x)| d x \\
& \leq C \int_{B\left(x_{0}, r_{0}\right)}\left(\int_{B\left(x_{0}, r_{0}\right)} \frac{|\nabla u(y)|^{\alpha}}{|x-y|^{n-\alpha} \varphi(|x-y|)} d y\right)|V(x)| d x \\
& =C \int_{B\left(x_{0}, r_{0}\right)}|\nabla u(y)|^{\alpha}\left(\int_{B\left(x_{0}, r_{0}\right)} \frac{|V(x)|}{|x-y|^{n-\alpha} \varphi(|x-y|)} d x\right) d y \\
& \leq C \int_{B\left(x_{0}, r_{0}\right)}|\nabla u(y)|^{\alpha}\left(\int_{B\left(y, 2 r_{0}\right)} \frac{|V(x)|}{|x-y|^{n-\alpha} \varphi(|x-y|)} d x\right) d y \\
& =C \xi_{V}\left(2 r_{0}\right) \int_{\mathbb{R}^{n}}|\nabla u(y)|^{\alpha} d y,
\end{aligned}
$$

with $C:=C\left(n, \alpha, \varphi, r_{0}\right)$.

By combining Lemma 3.1 and Theorem 3.2, we have the following corollary.

Corollary 3.3. Let $V \in S_{\alpha}, \frac{1}{\alpha}+\frac{1}{\alpha^{\prime}}=1$, and $\vartheta:=\frac{1}{\frac{\alpha^{\prime}}{\alpha}+1}$ such that

$$
\int_{0}^{1} \frac{\left[\eta_{\alpha} V(t)\right]^{1-\vartheta}}{t} d t<\infty
$$

Then

$$
\left\{\int_{\mathbb{R}^{n}}|u(x)|^{\alpha}|V(x)| d x\right\}^{\frac{1}{\alpha}} \leq C\left(n, \alpha, \eta_{\alpha} V, r_{0}\right)\|\nabla u\|_{L^{\alpha}}
$$

for every $u \in C_{0}^{\infty}\left(\mathbb{R}^{n}\right)$ with $\operatorname{supp}(u) \subseteq B\left(x_{0}, r_{0}\right)$.

Acknowledgement. This research is supported by ITB Research \& Innovation Program 2018.

\section{REFERENCES}

[1] D. Adams, "Traces of potentials arising from translation invariant operator", Ann. Scuola Norm. Sup. Pisa 25 (1971), 203-217.

[2] M. Aizenman and B. Simon, "Brownian motion and Harnack's inequality for Schrödinger operator", Comm. Pure. Appl. Math. 35 (1982), 209-273.

[3] R. E. Castillo, J. C. Ramos-Fernandes, and E. M. Rojas, "Properties of scales of Kato classes, Bessel potentials, Morrey spaces, and weak Harnack inequality for nonnegative solution of elliptic equations", J. Diff. Equat. 92 (2017), 1-17.

[4] F. Chiarenza, E. Fabes, and N. Garofalo, "Harnack's inequality for Schrödinger operators and the continuity of solutions", Proc. Amer. Math. Soc. 98 (1986), 415-425.

[5] E. B. Davis and A. Hinz, "Kato class potentials for higher order elliptic operators", J. London Math. Soc. 58 (1998), 669-678.

[6] G. Di Fazio, "Hölder continuity of solutions for some Schrödinger equations", Rend. Sem. Mat. Univ. Padova 79 (1988), 173-183. 
[7] Eridani and H. Gunawan, "Stummel classes and Morrey spaces", Southeast Asian Bull. Math. 29 (2005), 1053-1056.

[8] H. Gunawan, E. Nakai, Y. Sawano, and H. Tanaka, "Generalized Stummel class and Morrey spaces", Publ. Inst. Math. (New Series) 92(106) (2012), 127-138.

[9] A. Mohamed, "Weak Harnack's inequality for nonnegative solutions of elliptic equations with potential", Proc. Amer. Math. Soc. 129 (2001), 2617-2621.

[10] C. B. Morrey, "On the solutions of quasi-linear elliptic partial differential equations", Trans. Amer. Math. Soc. 43 (1938), 126-166.

[11] M. A. Ragusa and P. Zamboni, "A potential theoretic inequality", Czech. Mat. J. 51 (2001), $55-56$.

[12] S. Samko, "Morrey spaces are closely embedded between vanishing Stummel class", Math. Ineq. Appl. 17 (2014), 627-639. 